\title{
Modificaciones en el crecimiento temprano de trigo (Triticum aestivum $L$.) en presencia de Azospirillum brasilense y de Pseudomonas psychrophila
}

\author{
Palmero, F., Hang, S. B., Bigatton, E. D., Lucini, E., Davidenco, V. y Díaz-Zorita, M.
}

DOI: $10.31047 / 1668.298 x \cdot v 37 . n 1.27564$

\begin{abstract}
RESUMEN
Azospirillum spp. y Pseudomonas spp. son rizobacterias promotoras del crecimiento vegetal que mejoran el crecimiento y la productividad de los cultivos. El objetivo de este trabajo fue comparar los efectos de Azospirillum brasilense y de Pseudomonas psychrophila sobre el crecimiento inicial de trigo según condiciones contrastantes de fertilidad. El estudio se realizó en condiciones controladas de crecimiento combinando la inoculación con la fertilización con P y K en un sustrato estéril. Se evaluó el largo de raíces a los 10 días después de la siembra (DDS) y la acumulación de biomasa aérea y radicular a los 10 y a los 40 DDS. A los 10 DDS los tratamientos inoculados presentaron en promedio un aumento del $8 \%$ en el largo de las radículas, mientras que la biomasa total aumentó $6,3 \%$ al inocular con $A$. brasilense y $4,9 \%$ al inocular con P. psychrophila. A los 40 DDS, ambas rizobacterias aumentaron el crecimiento de las raíces en $21 \%$. En ambos momentos de evaluación, la acumulación de biomasa fue independiente de la fertilidad del sustrato, lo que sugiere que la presencia de A. brasilense y de P. psychrophila fue relevante durante la implantación y el crecimiento inicial del cultivo.
\end{abstract}

Palabras clave: biomasa; bioinsumos; nutrición mineral; rizobacterias promotoras del crecimiento vegetal; fósforo; potasio.

Palmero, F., Hang, S. B., Bigatton, E. D., Lucini, E., Davidenco, V. and Díaz-Zorita, M. 2020. Early wheat growth (Triticum aestivum L.) modifications in the presence of Azospirillum brasilense and Pseudomonas psychrophila. Agriscientia 37: 53-62

\section{SUMMARY}

Azospirillum spp. and Pseudomonas spp. are plant growth promoting rizobacteria that enhance crop growth and productivity. The aim of this article was to compare the effects of Azospirillum brasilense and Pseudomonas psychrophila over early wheat growth in contrasted fertility conditions. This research was carried out under controlled growth conditions combining 
inoculation and fertility with $\mathrm{P}$ and $\mathrm{K}$ in sterile substrate. Root length 10 days after sowing (DAS) and above ground and root biomass accumulation after 10 and 40 DAS were evaluated. 10 DAS, when inoculated, radicle length increased on average $8 \%$, while total biomass increased $6.3 \%$ when inoculated with A. brasilense and $4.9 \%$ when inoculated with P. psychrophila. 40 DAS, both rizobacteria increased root growth in $21 \% .10$ DAS and 40 DAS, biomass accumulation was independent from substrate fertility, suggesting that the presence of $A$. brasilense and $P$. psychrophila are relevant during implantation and early crop growth.

Key Words: biomass; bioinputs; mineral nutrition; plant growth-promoting rizobacteria; potassium; phosphorus.

Palmero, F. (ORCID: 0000-0003-0550-6003), Hang, S. (ORCID: 00000003-4017-7057), Bigatton, E. (ORCID: 0000-0002-7896-5369), Lucini, E. (ORCID: 0000-0003-0385-3271) y Davidenco, V. (ORCID: 00000002-4444-7977): Facultad de Ciencias Agropecuarias, Universidad Nacional de Córdoba. Félix Marrone 746, Ciudad Universitaria, Córdoba, Argentina. Díaz-Zorita, M. (ORCID: 0000-0003-3260-360X): Facultad de Agronomía, Universidad Nacional de La Pampa. Ruta Nacional 35, km 334, CP 6300, Santa Rosa, La Pampa, Argentina, y DZD Agro SRL. San Martín 430, CP 6237, Buenos Aires, Argentina. Correspondencia a: franciscopalmero@agro.unc.edu.ar

\section{INTRODUCCIÓN}

El trigo (Triticum aestivum L.), junto con el maíz (Zea mays L.) y el arroz (Oriza sativa L.), es uno de los cereales que más se cultiva en el mundo. En Occidente, el trigo es el cereal más consumido (Cuniberti, 2019), y en Argentina, ocupa el tercer lugar en toneladas de granos producidas después de la soja [Glycine max (L.) Merrill] y del maíz (FAOSTAT, 2018). Además de su importancia productiva, el trigo contribuye a mejorar condiciones físicas de los suelos dada la estructura de su sistema de raíces y su aporte de residuos orgánicos. Esto contribuye favorablemente a parámetros del suelo tales como la fertilidad, la estructura, la capacidad de intercambio catiónico y la capacidad de retención de agua (Wang, Fonte, Parikh, Six y Scow, 2017; Zhao et al., 2018).

En las condiciones extensivas predominantes en Argentina, la producción de trigo de، altos rendimientos requiere de la frecuente y abundante aplicación de nutrientes tales como nitrógeno (N) y fósforo (P) (García y Díaz-Zorita, 2015). Algunos de los riesgos del uso en exceso de $\mathrm{N}$ es su posibilidad de acumulación fuera del sitio de aplicación y la contaminación del medio ambiente
(Ni y Wang, 2015). Por eso, es importante plantear alternativas de manejo eficiente de los nutrientes, incorporando buenas prácticas de producción (Grasso y Díaz-Zorita, 2018). Una alternativa es el uso de rizobacterias promotoras del crecimiento vegetal (PGPR, según sus siglas en inglés). Estas bacterias inducen el crecimiento vegetal en forma directa o indirecta. En general, la influencia directa incluye la producción de fitohormonas, la solubilización de fosfatos y de micronutrientes y la fijación biológica de nitrógeno (Ruzzi y Aroca, 2015; Kumar, Singh, Tripathi, Singh y Singh, 2018). Los efectos indirectos resultan de la modificación del ambiente rizosférico debido a la solubilización de fósforo, y a la acción de las bacterias como biocontroladores de fitopatógenos mediante la liberación de sustancias como sideróforos, compuestos orgánicos volátiles (COVs), enzimas hidrolíticas, antibióticos y cianidas (Kumar et al., 2018).

Azospirillum spp. y Pseudomonas spp., reconocidos microrganismos PGPR con diferentes mecanismos de acción, han mostrado mejoras en el crecimiento y en la productividad de cultivos en diversas condiciones agroecológicas de producción (Saubidet, Fatta y Barneix, 2002; Díaz- 
Zorita y Fernández Canigia, 2009; Mehnaz, Kowalik, Reynolds y Lazarovits, 2010; Lana, Dartora, Marini y Hann, 2012; Puente et al., 2018; Goswami y Deka, 2020). Estas PGPR promueven mejoras en algunos parámetros relacionados a la germinación de las semillas y al crecimiento de las plántulas (Pathak, Chakrabarti, Das y Mandal, 2016; Safari, Jamali, Nooryazdan y Bayat, 2018; Yarzábal, Monserrate, Buela y Chica, 2018). También hay estudios que muestran que las plantas asociadas a las PGPR son afectadas en menor frecuencia por condiciones adversas tales como estrés hídrico, salino y térmico (Meena, Md. Afjal y Prakash, 2015; Safari et al., 2018; Azadikhah, Jamali, Nooryazdan y Bayat, 2019; Goswami y Deka, 2020). En particular, ante condiciones limitantes en la oferta normal de nutrientes, se ha observado que estos microorganismos mejoran la eficiencia de uso de los recursos disponibles en el suelo (Cassán y Díaz-Zorita, 2016).

El género Azospirillum está constituido por bacterias mutualistas no específicas capaces de habitar en la rizósfera de muchas especies vegetales. Promueven el crecimiento de las plantas por una sinergia de mecanismos tales como la producción de fitohormonas, la fijación de N, y la producción de sideróforos y de óxido nítrico (Cassán y García de Salamone, 2008; Bashan y de-Bashan, 2010; Lana et al., 2012; Mehnaz, 2015; Cassán y Díaz-Zorita, 2016; Coniglio, Mora, Puente y Cassán, 2019).

En la producción de trigo la inoculación con Azospirillum spp. es una práctica económicamente conveniente y se aplica principalmente como tratamiento a las semillas (Mehnaz et al., 2010). Al inocular con Azospirillum spp., se observan mayores rendimientos y contenidos de proteína en grano, atribuidos a un incremento en la absorción radical de N (Saubidet et al., 2002). El mayor crecimiento inicial de las raíces contribuye a mejorar la eficiencia de uso del agua y el aprovechamiento de las fuentes de nutrientes de las que disponen las plantas (Cassán y Díaz-Zorita, 2016). Además, la inoculación con Azospirillum spp. en la siembra de trigo, muestra aumentos en el número y en el largo de pelos radicales, en el número y en el diámetro de raíces laterales, en el peso fresco y seco de las raíces y de la parte aérea y en el rendimiento en granos (Díaz-Zorita y Fernández Canigia, 2009; Veresoglou y Menexes, 2010; Díaz-Zorita, Canigia, Bravo, Berger, y Satorre, 2015; Mehnaz, 2015). Los cambios en el crecimiento de las raíces se asocian principalmente a la producción de auxinas como producto del metabolismo de esta rizobacteria (Mehnaz, 2015). Las respuestas en los parámetros de crecimiento y de producción de trigo cuando se usan estas rizobacterias son variables. Se ha descrito que algunos de los factores que explican esta variabilidad se relacionan con el cultivar de trigo utilizado, la cepa de Azospirillum spp. aplicada y la cantidad de recursos disponibles que tienen las plantas para su crecimiento (Veresoglou y Menexes, 2010; Díaz-Zorita et al., 2015; Cassán y Díaz-Zorita, 2016; Pathak et al., 2016; Ludwig et al., 2018).

En presencia de Pseudomonas spp. se puede observar tanto el aumento del contenido de $\mathrm{N}$ y la acumulación de biomasa en tejidos vegetativos y reproductivos debido a aumentos en la absorción de $\mathrm{N}$, como el crecimiento del sistema radical debido a la producción de fitohormonas por parte de estas PGPR (Fox et al., 2016; Kumar et al. 2018). Se reconoce que estas PGPR contribuyen a incrementos en el crecimiento y en el rendimiento del trigo debido a mejoras en la solubilización del fósforo y del zinc del suelo al liberar ácidos orgánicos y fosfatasas que modifican el $\mathrm{pH}$ en la rizósfera (Oteino et al., 2015; Sirohi, Upadhyay, Srivastava y Srivastava, 2015; Rehman, Farooq, Naveed, Ozturk, y Nawaz, 2018; Liu et al., 2019). También Pseudomonas spp. promueven el crecimiento vegetal en forma indirecta actuando como agentes de biocontrol que protegen los vegetales del ataque de algunos microorganismos patógenos como Fusarium oxysporum, Pythium ultimum y Phytophtora infestans (Yarzábal et al., 2018). Estas PGPR actúan como biocontroladores mediante la inducción de resistencia sistémica en las plantas por la liberación de COVs y sideróforos, además de la liberación de antibióticos (Yuttavanichakul et al., 2012; Kejela, Thakkar y Patel, 2017).

La contribución de Azospirillum spp. y de Pseudomonas spp. sobre el crecimiento de trigo ha sido ampliamente documentada en estudios bajo condiciones controladas y extensivas de producción. Sin embargo, son escasos los estudios comparativos entre ambos microorganismos con el fin de discriminar sus aportes específicos durante la implantación y crecimiento del cultivo en condiciones contrastantes de disponibilidad de $\mathrm{P}$ y de K. Se espera que los cambios en plantas de trigo resultantes de la actividad directa de $A$. brasilense sean de mayor magnitud y se extiendan durante más etapas del crecimiento que el derivado de la actividad de P. psychrophila. Además, dada la contribución específica en la mejora de la disponibilidad de P, el mayor crecimiento al inocular con P. psychrophila se observaría solo en ausencia de correcciones por aplicación de nutrientes. 


\section{MATERIALES Y MÉTODOS}

\section{Microorganismos}

Se utilizaron como rizobacterias promotoras del crecimiento vegetal las especies $A$. brasilense cepa Az39 y P. psychrophila cepa PSE10 provistas por el cepario del laboratorio de Microbiología Agrícola de la Facultad de Ciencias Agropecuarias de la Universidad Nacional de Córdoba (Córdoba, Argentina). Los inoculantes se prepararon a partir del cultivo de $A$. brasilense en el medio descrito en la Tabla 1 y de P. psychrophila en Tripteína Soya Caldo (Britania B0210305 B0210306) a $28-30{ }^{\circ} \mathrm{C}$ durante

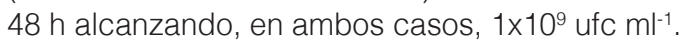

\section{Ensayo de germinación y de crecimiento en cámara de crecimiento}

Se estudió la germinación y el crecimiento inicial de trigo variedad BIOINTA 1006 en un sustrato de suelo y arena (Tabla 2) esterilizado y mantenido al $50 \%$ de su capacidad de campo (CC). La esterilización del sustrato fue realizada en autoclave durante $2 \mathrm{~h}$ a $121{ }^{\circ} \mathrm{C}$ y $1,21 \mathrm{kgF} \mathrm{cm}^{-2} \mathrm{de}$ presión.

Para determinar la CC se secó una masa de sustrato en estufa hasta peso constante (PS), luego se saturó con agua y se la dejó drenar hasta que se detuvo el flujo vertical, y se tomó el peso húmedo $(\mathrm{PH})$. El contenido de agua gravimétrico en CC se calculó según la siguiente ecuación,

$$
\mathrm{CC}(\%)=\left[(\mathrm{PH}-\mathrm{PS}) \mathrm{PS}^{-1}\right] \times 100 .
$$

Tabla 1. Composición del medio de cultivo para la preparación del inoculante con Azospirillum. El pH del medio se ajusta a 7 con $\mathrm{KOH} \mathrm{0,1} \mathrm{N}$.

\begin{tabular}{lc}
\hline $\mathrm{K}_{2} \mathrm{HPO}_{4}$ & $0,5 \mathrm{~g}$ \\
\hline $\mathrm{MgSO}_{4} 7 \mathrm{H}_{2} \mathrm{O}$ & $0,2 \mathrm{~g}$ \\
$\mathrm{NaCl}$ & $0,1 \mathrm{~g}$ \\
Extracto de levadura & $0,5 \mathrm{~g}$ \\
$\mathrm{FeCl}_{3} 6 \mathrm{H}_{2} \mathrm{O}$ & $0,015 \mathrm{~g}$ \\
$\mathrm{DL}$-ácido málico & $5 \mathrm{~g}$ \\
$\mathrm{KOH}$ & $4,8 \mathrm{~g}$ \\
$\mathrm{H}_{2} \mathrm{O}$ & $1000 \mathrm{ml}$ \\
\hline
\end{tabular}

Tabla 2. Caracterización química del sustrato utilizado, suelo:arena $(1: 1)$. Determinaciones previas a su esterilización. MO: materia orgánica, Nt: nitrógeno total, P-Bray: fosforo extractable según el

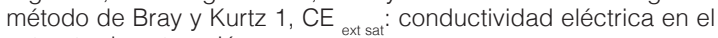
extracto de saturación

\begin{tabular}{|c|c|c|c|c|c|c|c|}
\hline nO & $\mathrm{Nt}$ & \multirow[t]{2}{*}{ C:N } & \multicolumn{3}{|c|}{$\mathrm{N}-\mathrm{NO}_{3}-\mathrm{S}_{-} \mathrm{SO}_{4}{ }^{2-} \mathrm{P}-\mathrm{Bray}$} & & \multirow{2}{*}{$\frac{\mathrm{CE}_{\text {ext.sat }}}{\mathrm{dS} \mathrm{m}^{-1}}$} \\
\hline \multicolumn{2}{|r|}{ 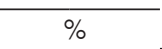 } & & & $\mathrm{mg} \mathrm{kg}^{-1}$ & & & \\
\hline 1,46 & 0,071 & 11,8 & 21,8 & 30,8 & 42,5 & 7, & 1,8 \\
\hline
\end{tabular}

Los tratamientos evaluados fueron: control sin inoculación ni fertilización (T0), fertilización con P y con K (T1), inoculación con A. brasilense (T2), inoculación con P. psychrophila (T3), inoculación con $A$. brasilense y fertilización con P y con K (T4) e inoculación con P. psychrophila y fertilización con $\mathrm{P}$ y con K (T5). Antes de su siembra, las semillas se desinfectaron con hipoclorito de sodio al $10 \%$ y luego se enjuagaron con agua destilada (Camiletti et al., 2018). En los tratamientos inoculados (T2 a T5), se aplicaron $80 \mu \mathrm{L}_{\text {semilla }}{ }^{-1}$ de los cultivos de microorganismos así alcanzaron un aporte aparente de $8 \times 10^{7}$ ufc semilla ${ }^{-1}$. Este tratamiento se realizó aplicando el $50 \%$ de la dosis sobre el sustrato en el punto de siembra de las semillas y el resto sobre las semillas luego de colocadas en el sustrato de crecimiento. Los tratamientos de fertilización (T1, T4 y T5) se realizaron con una solución con fosfato monobásico de potasio $\left(\mathrm{KH}_{2} \mathrm{PO}_{4}\right.$; Cogliatti, Cataldi e Iglesias, 2010) aplicado antes de la siembra en una dosis equivalente a $20 \mathrm{~kg} \mathrm{P} \mathrm{ha}^{-1}$ y a $25 \mathrm{~kg} \mathrm{~K} \mathrm{ha}^{-1}$ y en cada riego de mantenimiento hasta el $50 \%$ de CC del sustrato. Los controles sin fertilización (T0, T1 y T2) solo se regaron con agua destilada.

A los cuatro DDS se realizó el primer conteo de plántulas emergidas, y a los ocho DDS, el conteo final (Ludwig et al., 2018; ISTA 2020). A los diez DDS, se midió el largo de las radículas, el peso seco aéreo y el peso seco de las radículas. Se calculó el peso total de las plántulas a partir de la suma de los pesos secos aéreos y de las radículas. Para determinar el largo de raíces, se tomó como referencia el pelo radical de mayor extensión, a partir del pericarpio de cada semilla. El secado del material se realizó en estufa a $60{ }^{\circ} \mathrm{C}$ hasta peso constante, $48 \mathrm{~h}$ aproximadamente.

Dentro de la cámara, las unidades experimentales se distribuyeron al azar y se mantuvieron condiciones de $12 \mathrm{~h}$ de luz y $12 \mathrm{~h}$ de oscuridad (Safari. et al. 2018; ISTA 2020) y una temperatura de $22{ }^{\circ} \mathrm{C}$ durante 10 días. El diseño experimental fue bifactorial con un factor fertilidad y un factor PGPR. Se realizaron 3 repeticiones por tratamiento y se sembraron 20 semillas por bandeja. Con los resultados, se realizó un análisis de la varianza (ANAVA) utilizando el software InfoStat (Di Rienzo et al., 2014). Donde hubo diferencias significativas, se realizaron diferencias de medias usando la prueba DGC (Di Rienzo, Guzmán y Casanoves, 2002) con un nivel de significancia de 0,1.

\section{Ensayo de crecimiento en invernadero}

Se estudió el crecimiento de trigo variedad BIOINTA 1006 cultivado en macetas. Se utilizaron 
macetas de $815 \mathrm{~cm}^{3}$ lavadas con detergente y desinfectadas con solución de etanol al $70 \%$. El sustrato utilizado fue suelo y arena (1:1) (Tabla 2), que fue esterilizado y mantenido entre 60 y $80 \%$ de su CC a fin de facilitar la potencial expresión de desempeño de las PGPR (Skopp, Jawson y Doran 1990; Grant y Rochette 1994). Las plantas crecieron con luz natural en los meses de septiembre y octubre de 2019, y con una temperatura media de $23^{\circ} \mathrm{C}$ y $51 \%$ de humedad relativa. Se sembraron ocho semillas por maceta, y a los ocho DDS se raleó dejando seis plantas por maceta. Se realizaron siete repeticiones por tratamiento, siendo cada maceta una unidad experimental distribuida al azar. Los tratamientos (T0 a T5) y su instalación (esterilización de las semillas, inoculación, fertilización) se realizaron según lo descrito en el estudio en condiciones de cámara de crecimiento. El control y el mantenimiento del contenido de humedad de las macetas, se llevó a cabo por gravimetría, pesando y completando en cada maceta la diferencia de peso, producto de la transpiración y evaporación del sistema sueloplanta. Simultáneamente se registró la fenología de las plantas según Zadoks, Chang y Konzak (1974) y alcanzaron el estadio Z 15 al final del estudio (40 DDS).

A los 40 DDS se determinó el peso seco total (PST), el peso seco aéreo (PSA) y el peso seco de raíces (PSR) secando las plantas a $60^{\circ} \mathrm{C}$ hasta peso constante, aproximadamente durante 48 h. Además, se calculó un índice de partición de biomasa (IPB) como la relación entre el PSR y el PST de cada planta según la siguiente ecuación,

\section{IPB $=\left(\right.$ PSR planta $\left.{ }^{-1}\right) \times\left(\text { PST planta }{ }^{-1}\right)^{-1}$}

A los 40 DDS se determinó el área foliar verde de cada planta utilizando un integrador de área foliar LI 3000 (Li Cor, Inc., Lincoln, Nebraska, USA).

En ambos ensayos, los datos se analizaron con análisis de la varianza (ANAVA) utilizando el software InfoStat (Di Rienzo et al., 2014). Donde hubo diferencias significativas, se realizaron diferencias de medias usando el test DGC (Di Rienzo, Guzmán y Casanoves, 2002) con un nivel de significancia de 0,1.

\section{RESULTADOS Y DISCUSIÓN}

\section{Crecimiento de plántulas}

El largo de las raíces a los 10 DDS varió entre 13,2 y $27,7 \mathrm{~cm}$ planta $^{-1}$ (Tabla 3 ), con una media general de $20,4 \mathrm{~cm}$ entre todos los tratamientos mostró interacción significativa entre los factores fertilización e inoculación $(p>0,1)$. A. brasilense y $P$. psychrophila promovieron el crecimiento en longitud de las raíces de plántulas de trigo en etapas tempranas del crecimiento y las plantas inoculadas sin la incorporación de $\mathrm{P}$ y de $\mathrm{K}$ fueron las de mayor longitud (Tabla 3). Esto coincide con los resultados encontrados por Pathak et al. (2016), quienes inocularon con Azospirillum semillas de trigo que tenían distintos períodos de almacenamiento. Además, Safari et al. (2018) y Yarzábal et al. (2018) también mostraron que la inoculación con Pseudomonas spp. aumenta la longitud de las raíces en condiciones normales y de estrés por salinidad y por bajas temperaturas. En nuestro estudio, el efecto promotor del crecimiento de $A$. brasilense y $P$. psychrophila sobre el largo de raíces fue más notorio cuando estas rizobacterias se inocularon sin el agregado de P-K. Mientras que, al inocular en combinación con estos nutrientes, la longitud de las raíces fue similar a la alcanzada con solo el agregado de P-K. En los primeros 10 DDS el efecto promotor del crecimiento de estos organismos fue de mayor magnitud relativa en un sustrato limitado en $P$ y en $\mathrm{K}$, esto se debe presumiblemente a una mayor contribución sobre la actividad fisiológica de las plantas en comparación con un aumento en el acceso a nutrientes.

Tabla 3. Longitud de raíces de trigo evaluada a los 10 días de su siembra en un sustrato esterilizado según tratamientos de inoculación con Azospirillum y con Pseudomonas. Medias con letras distintas tienen diferencias significativas para un análisis DGC (Di Rienzo, et al., 2002) con $\mathrm{p}>0,10$. DE: desvío estándar de la media.

\begin{tabular}{|c|c|c|c|c|}
\hline \multicolumn{2}{|c|}{ Tratamiento (descripción) } & Media $\pm \mathrm{DE}$ & Mínimo & Máximo \\
\hline & & \multicolumn{3}{|c|}{$\mathrm{cm}_{\text {planta-1 }}^{-1}$} \\
\hline$\overline{\mathrm{TO}}$ & Control sin inocular ni fertilizar & $19,2 \pm 2,1(\mathrm{a})$ & 13,2 & 22,3 \\
\hline $\mathrm{T} 1$ & Fertilizado (PK) & $20,1 \pm 2,8(a)$ & 14,6 & 27,5 \\
\hline $\mathrm{T} 2$ & Inoculado con A. brasilense & $21,4 \pm 2,7(b)$ & 17,6 & 26,6 \\
\hline T3 & Inoculado con P. psychrophila & $21,3 \pm 2,5(b)$ & 15,7 & 26,3 \\
\hline $\mathrm{T} 4$ & Inoculado con A. brasilense + PK & $20,3 \pm 3,2(a)$ & 13,8 & 27,7 \\
\hline $\mathrm{T} 5$ & Inoculado con P. psychrophila + PK & $20,0 \pm 2,9(a)$ & 13,6 & 25,6 \\
\hline
\end{tabular}




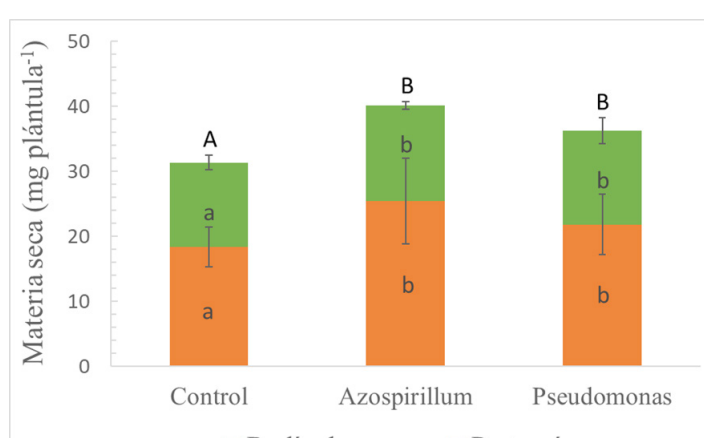

- Radículas $\quad$ Parte aérea

Figura 1. Materia seca de plántulas de trigo acumulada a los 10 días de la siembra según tratamientos de inoculación con $A$. brasilense y con P. psychrophila. Promedio de tratamientos con y sin fertilización con $\mathrm{P}$ y con $\mathrm{K}$. Letras minúsculas diferentes indican diferencias en la materia seca de las radículas o de la parte aérea entre tratamientos de inoculación $(p>0,10)$. Letras mayúsculas diferentes muestran diferencias en la acumulación total de materia seca entre tratamientos de inoculación $(p>0,10)$. Las barras muestran el desvío estándar de las medias.

La biomasa total alcanzada a los 10 DDS en las plántulas tratadas con $A$. brasilense fue 6,3\% mayor que en el control sin inocular, mientras que las plántulas tratadas con P. psychrophila lo superaron en $4,9 \%$ (Figura 1). No obstante, las diferencias entre ambos microorganismos no fueron significativas. El factor fertilidad a los 10 DDS no tuvo un efecto significativo en el crecimiento de las plántulas de trigo y no se detectó interacción entre los factores fertilidad y PGPR $(p>0,1)$.

Al particionar la biomasa total en biomasa aérea y radicular, se observó que los efectos de la inoculación con una u otra rizobacteria fueron independientes del efecto de fertilización con $\mathrm{P}$ y con K (interacción Fertilización x Inoculación no significativa, $p>0,1)$. En la Figura 1, se observa que la inoculación con los PGPR incrementó el crecimiento aéreo de las plántulas. Al igual que para la biomasa total, la biomasa aérea se incrementó en promedio $13 \%$ sobre el control al inocular con $A$. brasilense (14,7 mg plántula $\left.{ }^{-1}\right)$ o con P. psychrophila (14,5 mg plántula-1). En contraposición, Liu et al. (2019) evaluaron el crecimiento de plantas de trigo inoculadas con Pseudomonas en condiciones no controladas en bandejas para crecimiento de raíces y no observaron modificaciones en la biomasa total y de raíces a los 6 y a los 12 días desde su siembra con respecto al control. En nuestro estudio, a los 10 DDS, la biomasa de las raíces fue la variable de respuesta que mejor mostró los aportes de los PGPR sobre el crecimiento de plántulas de trigo (Figura 1). Al agregar A. brasilense, el peso seco de las radículas fue de $25,5 \mathrm{mg}$ plántula ${ }^{-1}$, mientras que el alcanzado con P. psychrophila fue de 21,8 mg plántula ${ }^{-1}$. Este resultado con $A$. brasilense sugiere efectos hormonales de estas bacterias sobre las plántulas que conducen a cambios en los patrones de crecimiento al aumentar la partición de asimilados hacia las raíces. Esto se explica, en parte, por el efecto PGPR de Azospirillum asociado principalmente a la producción de fitohormonas o compuestos precursores de estos (Perrig et al., 2007; Mehnaz, 2015). En tanto que el efecto de Pseudomonas se debe principalmente a aportes indirectos a partir de mejoras en la solubilización del P inorgánico (Oteino et al., 2015).

\section{Ensayo de crecimiento en condiciones de invernadero}

Los resultados del ensayo de crecimiento de trigo para biomasa total y particionada en aérea y radicular evaluada a los 40 DDS se presentan en la Tabla 4. No se detectó interacción significativa entre los factores inoculación con PGPR y fertilización con P-K $(p>0,1)$, pero sí se identificaron

Tabla 4. Producción de materia seca de trigo a los 40 días después de la siembra según dos niveles de fertilización con $\mathrm{P}$ y con $\mathrm{K}$ e inoculación con Azospirillum y con Pseudomonas.

\begin{tabular}{lccc}
\hline Análisis de la varianza & Peso Seco Total & Peso Seco Aéreo Peso Seco de Raíces \\
\hline Fertilización & 0,0001 & 0,0002 & 0,0002 \\
Inoculación & 0,0134 & $\mathrm{~ns}$ & 0,0008 \\
Fertilización x Inoculación & $\mathrm{ns}$ & $\mathrm{ns}$ & $\mathrm{ns}$ \\
Fertilización & & $\mathrm{g}$ planta-1 & $0,116 \mathrm{a}$ \\
Control sin PK & $0,243 \mathrm{a}$ & $0,127 \mathrm{a}$ & $0,147 \mathrm{~b}$ \\
Fertilizado con PK & $0,307 \mathrm{~b}$ & $0,160 \mathrm{~b}$ & $0,113 \mathrm{a}$ \\
Inoculación & & & $0,136 \mathrm{~b}$ \\
$\quad$ Control sin inocular & $0,254 \mathrm{a}$ & $0,140 \mathrm{a}$ & $0,138 \mathrm{~b}$ \\
$\quad$ Inoculado con A. brasilense & $0,273 \mathrm{~b}$ & $0,137 \mathrm{a}$ & $0,148 \mathrm{a}$ \\
\hline
\end{tabular}

Medias con letras distintas tienen diferencias significativas para un análisis DGC con $p>0,10$. 
respuestas a la aplicación de los nutrientes y de los microorganismos de forma independiente. En promedio no se observaron diferencias en estos parámetros entre los PGPR.

El peso seco de las raíces mostró efectos independientes entre los tratamientos de fertilización con P y con K y de inoculación $(p>0,1)$. La inoculación con $A$. brasilense contribuyó a incrementos de la biomasa radical de $20,3 \%$ con respecto al control. Estas respuestas coinciden con lo reportado por Díaz-Zorita y Fernández Canigia (2009) que mostraron aumentos del $22 \%$ en el peso seco de raíces al estudiar la inoculación de trigo con A. brasilense en 297 sitios experimentales bajo condiciones de producción en el campo en la región pampeana de Argentina. La inoculación con P. psychrophila condujo a aumentos del $22,1 \%$ en el peso seco de raíces con respecto al control. En concordancia con estos resultados, Meena et al. (2015) también observaron incrementos en la biomasa radical de plantas de trigo inoculadas con $P$. aeruginosa en condiciones controladas simulando estrés por temperatura elevada. En nuestro estudio, los incrementos de biomasa total y radical de los tratamientos fertilizados e inoculados respecto al control se observaron a pesar de las condiciones de estrés hídrico moderadas impuestas durante el desarrollo de las plantas (60 - $80 \%$ CC del sustrato).

A medida que el trigo avanzó en su fenología, aumentó la importancia del efecto de la aplicación de nutrientes, ya que el aporte de Py de Kincrementó la biomasa total en 26,3\% en comparación con los controles sin fertilización (Tabla 4). El peso seco

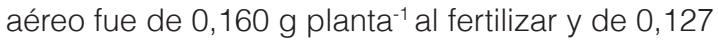
g planta ${ }^{-1}$ sin el agregado de P y de K. Similares resultados al fertilizar se observaron sobre la acumulación de biomasa de las raíces.

La inoculación con las PGPR no mostró cambios significativos en el crecimiento aéreo. Sin embargo, la inoculación con P. psychrophila mostró una tendencia a ser el tratamiento PGPR con mayor biomasa aérea (Tabla 4). Las posibles diferencias en respuestas entre la inoculación con $A$. brasilense y con P. psychrophila podría atribuirse parcialmente por el $\mathrm{pH}$ del sustrato que, al ser neutro, decrece la disponibilidad de P para las plantas (Thompson y Troeh, 1980). En un escenario con estas características es de mayor relevancia el efecto solubilizador de $P$ de Pseudomonas al liberar ácidos orgánicos (Liu et al., 2019). No obstante, Veresouglou y Menexes (2010), haciendo una revisión de publicaciones en las que se estudió el efecto de la inoculación con Azospirillum spp. sobre trigo, describieron aumentos medios del 17,8 \% en la biomasa aérea de plantas inoculadas con Azospirillum spp. En nuestro estudio esos resultados no fueron corroborados, probablemente debido a diferencias en los momentos de evaluación y a que las mejoras en acumulados en crecimiento aéreo se detecten con mayor consistencia en estadios fenológicos más avanzados.

Solo las PGPR modificaron la relación de partición de biomasa mostrando el mayor IPB cuando las semillas fueron inoculadas con $A$. brasilense (Tabla 5). Al inocular, la proporción de biomasa acumulada de raíces fue mayor que al no hacerlo e independientemente del agregado de nutrientes (Tabla 5). Los microorganismos PGPR producen fitohormonas, metabolitos y enzimas que inducen a modificaciones en la expresión de genes y en el patrón de crecimiento de las plantas (Fahad et al., 2014; Goswami y Deka, 2020). El mayor IPB alcanzado en los tratamientos con PGPR sugiere que la incorporación de estas bacterias genera efectos hormonales que conllevan a un incremento de la partición de asimilados hacia las raíces. Los cambios en la cantidad de nutrientes no alteraron la partición de fotoasimilados evaluada a partir de la relación entre la biomasa acumulada aérea y de raíces (Tabla 5). Tal como se observó en los estadios iniciales del crecimiento, los aportes sobre la biomasa de las raíces fueron de mayor magnitud en presencia de $A$. brasilense que con $P$. psychrophila (Figura 1).

La fertilización con $\mathrm{P}$ y con $\mathrm{K}$, independientemente de la inoculación, incrementó el área foliar fotosintéticamente activa de las plantas evaluada a los 40 DDS (Tabla 5). Esta variable fue 14,2 \% mayor al fertilizar que en el control sin aplicación de $P$ y de $K$ (Figura 3). En este estudio no se

Tabla 5. Partición de biomasa seca y área foliar de trigo a los 40 días después la siembra con y sin fertilización con $\mathrm{P}$ y con $\mathrm{K}$ e inoculación con Azospirillum y con Pseudomonas.

\begin{tabular}{lcc}
\hline Análisis de la varianza & IPB & AF \\
\hline Fertilización & ns & 0,0023 \\
Inoculación & 0,0001 & ns \\
Fertilización x Inoculación & ns & ns \\
Fertilización & & cm² $^{2}$ planta $^{-1}$ \\
$\quad$ Control sin PK & 0,47 a & - \\
$\quad$ Fertilizado con PK & 0,48 a & - \\
Inoculación & & \\
Control sin inocular & - & 23,2 a \\
Inoculado con A. brasilense & - & 24,6 a \\
Inoculado con P. psychrophila & - & 25,8 a \\
\hline
\end{tabular}

Medias con letras distintas tienen diferencias significativas para un análisis DGC (Di Rienzo, Guzmán y Casanoves, 2002) con $p>0,1$. La ausencia de valores medios indican diferencias significativas entre tratamientos del factor evaluado. 


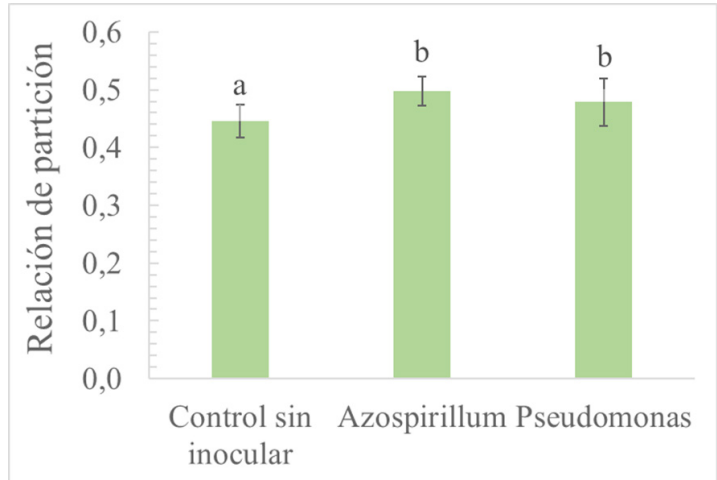

Figura 2. Partición de biomasa de trigo acumulada a los 40 días de la siembra según tratamientos de inoculación con $A$. brasilense y con P. psychrophila. Promedio de tratamientos con y sin fertilización con $\mathrm{P}$ y con K. Relación de partición: (peso seco de raíces) $\times$ (peso seco total) $)^{-1}$. Letras diferentes indican diferencias entre tratamientos de inoculación $(p>0,10)$. Las barras muestran el desvío estándar de las medias.

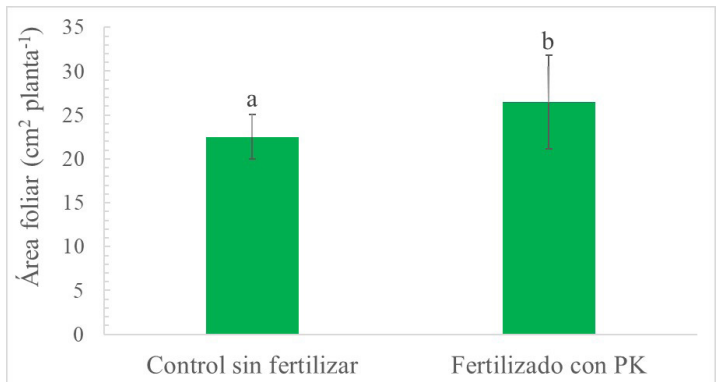

Figura 3. Área foliar de trigo a los 40 días de la siembra según tratamientos de fertilización con $\mathrm{P}$ y con $\mathrm{K}$. Promedio de tratamientos de inoculación con $A$. brasilense y con $P$. psychrophila. Letras diferentes indican diferencias entre tratamientos de fertilización $(p>0,10)$. Las barras muestran el desvío estándar de las medias.

encontraron efectos significativos de las PGPR sobre el área foliar, no obstante, Mazhar, llyas, Saeed, Bibi y Batool (2016) evaluaron el efecto de la inoculación con $A$. lipoferum sobre plantas de trigo en condiciones controladas, y encontraron aumentos de $8 \%$ en el área foliar en las plantas inoculadas con respecto al control, tanto en condiciones normales como en estrés salino. Meena et al. (2015) también mostraron aumentos en el área foliar de plantas de trigo que fueron inoculadas con $P$. aeruginosa en condiciones de estrés por altas temperaturas. Por lo tanto, no debe ser descartado el efecto de las PGPR sobre el área foliar en plantas de trigo, tanto en condiciones normales como de estrés durante el crecimiento de las plantas.

\section{CONCLUSIONES}

En condiciones controladas de crecimiento, se validó que la incorporación tanto de $A$. brasilense como de P. psychrophila mejora el crecimiento inicial del trigo al incrementar la elongación y la acumulación de biomasa de las raíces. Al inocular con $A$. brasilense se modificó la partición de biomasa hacia las raíces lo que sugiere un mayor efecto fitohormonal que por la presencia de $P$. psychrophila. Los tratamientos de inoculación mostraron efectos sobre la acumulación de biomasa total del trigo independientemente de la fertilización con $\mathrm{P}$ y con $\mathrm{K}$ lo que sugiere la ocurrencia de aportes combinados no compensatorios de ambos factores sobre el crecimiento.

\section{BIBLIOGRAFIA}

Azadikhah, M., Jamali, F., Nooryazdan, H. R. y Bayat, F. (2019). Growth promotion and yield enhancement of barley cultivars using ACC deaminase producing Pseudomonas fluorescens strains under salt stress. Spanish Journal of Agricultural Research, 17 (1). doi: 10.5424/sjar/2019171-13828

Bashan, Y. y de-Bashan, L. E. (2010). How the Plant Growth-Promoting Bacterium Azospirillum Promotes Plant Growth-A Critical Assessment. Advances in Agronomy, 108, 77-136. doi: 10.1016/s00652113(10)08002-8

Camiletti, B. X., Moral, J., Asensio, C. M., Torrico, A. K., Lucini, E. I., Giménez-Pecci, M. P. y Michailides, T. J. (2018). Characterization of Argentinian Endemic Aspergillus flavus Isolates and Their Potential Use as Biocontrol Agents for Mycotoxins in Maize. Phytopathology, 108 (7), 818-828. doi: 10.1094/phyto07-17-0255-r

Cassán, F. D. y García de Salamone, I. (2008). Azospirillum sp.: cell physiology, plant interactions and agronomic research in Argentina. Buenos Aires, Argentina: Asociación Argentina de Microbiología.

Cassán, F. y Diaz-Zorita, M. (2016). Azospirillum sp. in current agriculture: From the laboratory to the field. Soil Biology and Biochemistry, 103, 117-130. doi: 10.1016/j.soilbio.2016.08.020

Cogliatti, D.H., Cataldi, M.F. e Iglesias, F. (2010). Estimación del área de las hojas en plantas de trigo bajo diferentes tipos de estrés abiótico. Agriscientia, 2010, 27(1), 43-53. doi: 10.31047/1668.298x.v27. n1.2764

Coniglio A., Mora V., Puente M. y Cassán F. (2019) Azospirillum as Biofertilizer for Sustainable Agriculture: Azospirillum brasilense AZ39 as a Model of PGPR and Field Traceability. En D, Zúñiga-Dávila, F, González- 
Andrés y E Ormeño-Orrillo (Eds), Microbial Probiotics for Agricultural Systems. Sustainability in Plant and Crop Protection (45-70). Cham, Swizterland: Springer. doi: 10.1007/978-3-030-17597-9_4

Cuniberti, M. (2019). Producción de trigo en Argentina y el mundo. En A. E. León, G. Pérez y P. Ribotta (Eds.). Trigo: un cereal único (21- 52). Villa María, Argentina: Eduvim.

Di Rienzo, J. A., Guzmán A. W. y Casanoves F. (2002). A Multiple Comparisons Method based on the Distribution of the Root Node Distance of a Binary Tree. Journal of Agricultural, Biological, and Environment Statistics, 7 (2): 1-14.

Di Rienzo, J. A., Casanoves, F., Balzarini, M. G., Gonzalez, L., Tablada, M. y Robledo, C. W. Infostat (versión 2014) [Software de cómputo] Córdoba, Argentina: Grupo InfoStat, FCA, Universidad Nacional de Córdoba. URL: http://www.infostat.com.ar

Díaz-Zorita, M., Canigia, M. V. F., Bravo, O. Á., Berger, A. y Satorre, E. H. (2015). Field Evaluation of Extensive Crops Inoculated with Azospirillum sp. En F. D. Cassán, Y. Okon and C. M. Creus (Eds.) Handbook for Azospirillum (435-445). Cham, Swizterland: Springer. doi: 10.1007/978-3-319-06542-7_24

Díaz-Zorita, M. y Fernández-Canigia, M. V. (2009). Field performance of a liquid formulation of Azospirillum brasilense on dryland wheat productivity. European Journal of Soil Biology, 45 (1), 3-11. doi: 10.1016/j. ejsobi.2008.07.001

Fahad, S., Hussain, S., Bano, A., Saud, S., Hassan, S., Shan, D., ... Huang, J. (2014). Potential role of phytohormones and plant growth-promoting rhizobacteria in abiotic stresses: consequences for changing environment. Environmental Science and Pollution Research, 22 (7), 4907-4921. doi: 10.1007/ s11356-014-3754-2

FAOSTAT (2018). Organización de las Naciones Unidas para la Alimentación y la Agricultura, División de Estadística, Comparar datos. Recuperado de: http:// www.fao.org/faostat/es/\#compare

Fox, A. R., Soto, G., Valverde, C., Russo, D., Lagares, A., Zorreguieta, Á., ... Ayub, N. D. (2016). Major cereal crops benefit from biological nitrogen fixation when inoculated with the nitrogen-fixing bacterium Pseudomonas protegens Pf-5 X940. Environmental Microbiology, 18 (10), 3522-3534. doi: 10.1111/14622920.13376

García, F.O. y Díaz-Zorita, M. 2015. La fertilidad de los suelos y el uso de nutrientes en la producción agrícola extensiva de Argentina. En R.R. Casas y G.F. Albarracín (Eds.), El deterioro del suelo y del ambiente en la Argentina (183-198). Ciudad Autónoma de Buenos Aires, Argentina: FECIC.

Goswami, M. y Deka, S. (2020). Plant growth-promoting rhizobacteria-alleviators of abiotic stresses in soil: A review. Pedosphere, 30 (1), 40-61. doi: 10.1016/ s1002-0160(19)60839-8

Grant, R. F. y Rochette, P. (1994). Soil Microbial Respiration at Different Water Potentials and Temperatures: Theory and Mathematical Modeling. Soil Science Society of America Journal, 58 (6), 1681-1690. doi: 10.2136/ sssaj1994.03615995005800060015x

Grasso, A. A. y Díaz-Zorita, M. (2018). Manual de buenas prácticas de manejo de fertilización. [Libro digital] Recuperado de https://www.fertilizar.org.ar/subida/ BMPN/BPMN_Fertilizar102018.pdf

ISTA (International Seed Testing Association). (2020). International Rules for Seed Testing. Seed Science and Technology 31. Zürich, Switzzerland: ISTA. https:// doi.org/10.15258/istarules.2020.F

Kejela, T., Thakkar, V. R. y Patel, R. R. (2017). A novel strain of Pseudomonas inhibits Colletotrichum gloeosporioides and Fusarium oxysporum infections and promotes germination of coffee. Rhizosphere, 4, 9-15. doi: 10.1016/j.rhisph.2017.05.002

Kumar, A., Singh, V. K., Tripathi, V., Singh, P. P. y Singh, A. K. (2018). Plant Growth-Promoting Rhizobacteria (PGPR): Perspective in Agriculture Under Biotic and Abiotic Stress. En R. Prasad, S., Gill, S. y Tuteja, N. (Eds.), New and Future Developments in Microbial Biotechnology and Bioengeneering.Crop Improvement Through Microbial Biotechnology (333-342). [Libro electrónico]. Elsevier. doi: 10.1016/b978-0-444-639875.00016-5

Lana, M. do C., Dartora, J., Marini, D. y Hann, J. E. (2012). Inoculation with Azospirillum, associated with nitrogen fertilization in maize. Revista Ceres, 59 (3), 399-405. doi: 10.1590/s0034-737x2012000300016

Liu, X., Jiang, X., He, X., Zhao, W., Cao, Y., Guo, T., ... Tang, X. (2019). Phosphate-Solubilizing Pseudomonas sp. Strain P34-L Promotes Wheat Growth by Colonizing the Wheat Rhizosphere and Improving the Wheat Root System and Soil Phosphorus Nutritional Status. Journal of Plant Growth Regulation, 38, 1314-1324. doi: 10.1007/s00344-019-09935-8

Ludwig, R. L., Martin, T. N., Stecca, J. D. L., Cunha, V. dos S., Nunes, U. R. y Grando, L. F. T. (2018). Action specificity of chemical treatment and inoculation with Azospirillum brasilense in wheat seed on the crop initial growth. Revista Ceres, 65 (5), 407-414. doi: 10.1590/0034-737×201865050005

Mazhar, R., Ilyas, N., Saeed, M., Bibi, F. y Batool, N. (2016). Biocontrol and salinity tolerance potential of Azospirillum lipoferum and its inoculation effect in wheat crop. International Journal of Agriculture \& Biology, 18 (3), 494 500. doi: 10.17957/JJAB/15.0115

Meena, H., Ahmed, Md. A. y Prakash, P. (2015). Amelioration of heat stress in wheat, Triticum 
aestivum by PGPR (Pseudomonas aeruginosa strain 2CpS1). Bioscience. Biotechnology Research Communications, 8 (2): 171-174.

Mehnaz, S. (2015) Azospirillum: A Biofertilizer for Every Crop. En: N. K. Arora (Ed), Plant Microbes Symbiosis: Applied Facets, (297-314). New Delhi, India: Springer. doi: 10.1007/978-81-322-2068-8_15

Mehnaz, S., Kowalik, T., Reynolds, B. y Lazarovits, G. (2010). Growth promoting effects of corn (Zea mays) bacterial isolates under greenhouse and field conditions. Soil Biology and Biochemistry, 42 (10), 1848-1856. doi: 10.1016/j.soilbio.2010.07.003

$\mathrm{Ni}$, Z. y Wang, S. (2015). Historical accumulation and environmental risk of nitrogen and phosphorus in sediments of Erhai Lake, Southwest China. Ecological Engineering, 79, 42-53. doi: 10.1016/j. ecoleng.2015.03.005

Oteino, N., Lally, R. D., Kiwanuka, S., Lloyd, A., Ryan, D., Germaine, K. J. y Dowling, D. N. (2015). Plant growth promotion induced by phosphate solubilizing endophytic Pseudomonas isolates. Frontiers in Microbiology, 6, 745. doi: 10.3389/fmicb.2015.00745

Pathak, A., Chakrabarti, S. K., Das, R. y Mandal, M. K. (2016). Response of different wheat varieties towards Azospirillum and phosphate solubilizing bacteria (PSB) seed inoculation. Journal of Applied and Natural Science 8 (1), 213 - 217. doi: 10.31018/jans.v8i1.775

Perrig, D., Boiero, M. L., Masciarelli, O. A., Penna, C., Ruiz, O. A., Cassán, F. D. y Luna, M. V. (2007). Plant-growth-promoting compounds produced by two agronomically important strains of Azospirillum brasilense, and implications for inoculant formulation. Applied Microbiology and Biotechnology, 75 (5), 1143-1150. doi: 10.1007/s00253-007-0909-9

Puente, M. L., Zawoznik, M., de Sabando, M. L., Perez, G., Gualpa, J. L., Carletti, S. M. y Cassán, F. D. (2018). Improvement of soybean grain nutritional quality under foliar inoculation with Azospirillum brasilense strain Az39, Symbiosis. 77,41-47. doi: 10.1007/s13199-0180568-x

Rehman, A., Farooq, M., Naveed, M., Ozturk, L. y Nawaz, A. (2018). Pseudomonas-aided zinc application improves the productivity and biofortification of bread wheat. Crop and Pasture Science, 69 (7), 659-672. doi: 10.1071/cp17441

Ruzzi, M. y Aroca, R. (2015). Plant growth-promoting rhizobacteria act as biostimulants in horticulture. Scientia Horticulturae, 196, 124-134. doi: 10.1016/j. scienta.2015.08.042

Safari, D., Jamali, F., Nooryazdan, H. y Bayat, F. (2018). Evaluation of ACC deaminase producing Pseudomonas fluorescens strains for their effects on seed germination and early growth of wheat under salt stress. Australian Journal of Crop Science, 12 (3), 413-421. doi: 10.21475/ajcs. 18.12.03.pne801

Saubidet, M. I., Fatta, N. y Barneix, A. J. (2002). The effect of inoculation with Azospirillum brasilense on growth and nitrogen utilization by wheat plants. Plant and Soil, 245 (2), 215-222. doi:10.1023/a:1020469603941

Sirohi, G., Upadhyay, A., Srivastava, P. y Srivastava, S. (2015). PGPR mediated Zinc biofertilization of soil and its impact on growth and productivity of wheat. Journal of Soil Science and Plant Nutrition, 15 (1), 202-216. doi: 10.4067/s0718-95162015005000017

Skopp, J., Jawson, M. D. y Doran, J. W. (1990). SteadyState Aerobic Microbial Activity as a Function of Soil Water Content. Soil Science Society of America Journal, 54 (6), 1619-1625. doi: 10.2136/ sssaj1990.03615995005400060018x

Thompson, L.M. y Troeh, F.R. (1980). Los suelos y su Fertilidad (4a ed). Barcelona, España: Reverté

Veresoglou, S. D. y Menexes, G. (2010). Impact of inoculation with Azospirillum spp. on growth properties and seed yield of wheat: a meta-analysis of studies in the ISI Web of Science from 1981 to 2008. Plant and Soil, 337, 469-480. doi: 10.1007/s11104-010-0543-7

Wang, D., Fonte, S. J., Parikh, S. J., Six, J. y Scow, K. M. (2017). Biochar additions can enhance soil structure and the physical stabilization of $\mathrm{C}$ in aggregates. Geoderma, 303, 110-117. doi: 10.1016/j. geoderma.2017.05.027

Yarzábal, L. A., Monserrate, L., Buela, L. y Chica, E. (2018). Antarctic Pseudomonas spp. promote wheat germination and growth at low temperatures. Polar Biology, 41, 2343-2354. doi: 10.1007/s00300-0182374-6

Yuttavanichakul, W., Lawongsa, P., Wongkaew, S., Teaumroong, N., Boonkerd, N., Nomura, N. y Tittabutr, P. (2012). Improvement of peanut rhizobial inoculant by incorporation of plant growth promoting rhizobacteria (PGPR) as biocontrol against the seed borne fungus, Aspergillus niger. Biological Control, 63 (2), 87-97. doi: 10.1016/j.biocontrol.2012.06.008

Zadoks, J. C., Chang, T. T. y Konzak, C. F. (1974). A decimal code for the growth stages of cereals. Weed Research, 14 (6), 415-421. doi: 10.1111/j.13653180.1974.tb01084.x

Zhao, H., Shar, A. G., Li, S., Chen, Y., Shi, J., Zhang, X., y Tian, X. (2018). Effect of straw return mode on soil aggregation and aggregate carbon content in an annual maize-wheat double cropping system.Soil and Tillage Research, 175,178-186. doi:10.1016/j. still.2017.09.012 